\title{
SPECTRAL ANALYSIS OF THE WOLF-RAYET STAR HD 50896
}

\author{
W. -R. Hamann, W. Schmutz, U. Wessolowski \\ Institut für Theoretische Physik \\ und Sternwarte der Universität Kiel \\ Federal Republic of Germany
}

\section{INTRODDCTION}

The helium spectrum of the WN5 star HD 50896 (EZ Canis Majoris, WR6) is studied. Our aim is to establish a technique which allows the determination of the parameters of a Wolf-Rayet star from a systematic analysis of its spectral lines. Since the method of "iteration with approximate Lambda operators" became available for application to expanding atmospheres (Hamann, 1986, 1987), we are now able to compare observed spectra to realistic model calculations (Hamann and schmutz, 1987; Wessolowski et a.., 1987).

\section{THE THEORETICAL MODELS}

We consider the non-LTE spectral formation in a spherically expanding atmosphere. The velocity field $v(r)$ is specified in its supersonic part by the usual analytical law with the parameters $v_{\infty}$ (final velocity) and the exponent $B=1$. The temperature structure is derived from the assumption of radiative equilibrium, but only approximately evaluated for the grey LTE case. The atmosphere is assumed to consist of pure helium. The model atom has a total of 28 energy levels, among these 17 levels of He $I$. The line radiation transfer is treated in the "comoving frame".

\section{FIT OF THE OBSERVATIONS}

Three important parameters enter the model calculations: the mass loss rate $\dot{M}$, the stellar radius $R_{*}$ and the temperature parameter $T_{*}$. The fit of the helium line profiles of HD 50896 requires a final wind velocity of $1700 \mathrm{~km} / \mathrm{s}$. Hence, we now calculate a small grid of models in the appropriate range of $R_{\star}$ and $T_{*}$ and with the specially adapted $v_{\infty}$. The mass-loss rate is kept at $\log (\dot{M} /(M / y r))^{*}=-4.4$ as an arbitrary choice. The results are presented in the form of contour lines in the

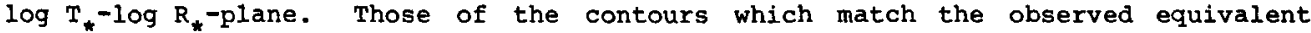
widths or peak intensities are extracted and yield a "fit diagram". We obtain a well-defined intersection region centered about $R_{*}=2.6 R_{*}, T_{*}=60 \mathrm{kK}$ (hereafter quoted as "model B").

As the mass-loss rate is is considered as a free parameter, we now repeat the whole fit procedure for different values, namely $\log (\dot{M} /(M / Y x))=-4.0$ (case " $A$ ") and -4.7 (case "C") (cf. Table 1). The resulting fit diagrams look qualitatively similar for all three mass-loss rates considered. There is no obvious preference in favour of a certain mass-loss rate from the internal consistency of the fits.

The obtained "fit point" parameters are now used for the calculation of "final models", and the resulting synthetic spectra are compared with the observation as a final check. We restrict the representation (Fig. 1) to the model $C$, since the corresponding profile fits for the other two mass-loss rates would appear very similar.

In total, we feel that the agreement between theoretical and observed line profiles is satisfactory and internally consistent. We take this as a confirmation of the basic physical input of our models, and as evidence that the results of the analysis are reliable. 
Table 1. Stellar parameters for different adopted mass-loss rates

\begin{tabular}{lrcc}
\hline Model & A & B & C \\
\hline $\left.\log \left(\dot{M} / \mathrm{M}_{\odot} / \mathrm{Yr}\right)\right)$ & -4.0 & -4.4 & -4.7 \\
$\mathrm{R}_{\star} / \mathrm{R}_{\odot}$ & 4.7 & 2.6 & 1.5 \\
$\mathrm{~T}_{\star} / \mathrm{kK}$ & 57.5 & 60 & 63 \\
$\log \left(\mathrm{L} / \mathrm{L}_{\odot}\right)$ & 5.3 & 4.9 & 4.5 \\
\hline
\end{tabular}

\section{RESULTS AND DISCUSSION}

The spectral analysis of HD 50896 performed in sect. 3 resulted in a one-dimensional set of solutions for the three parameters $M, R_{*}$ and $T_{*}$, i.e. one of these parameters (e.g. the mass-loss rate) is still free (cf. Table 1). A comparison with Hillier (1987) reveals that his results are comparable with our model B. A restriction to an unique solution would be possible if the distance of this star could be determined.

An independent determination for the distance of HD 50896 does not exist. The "possible membership" (Lundström and Stenholm, 1984) to Collinder 121 ( $\alpha=0.91 \mathrm{kpc}$ ) can be ruled out from a study of the interstellar Na I lines in the spectrum of HD 50896 and of neighbouring stars (Schmutz and Howarth, in preparation). We conclude that HD 50896 may have any distance between $0.91 \mathrm{kpc}$ and a few kiloparsecs, but there is some evidence for a value of about 2 kpc. The comparison between apparent and absolute magnitudes then leads to our model $A$, while the models $B$ and $C$ correspond to smaller distances.

The radio observations (Hogg, 1982) are in agreement with the results of our analysis, but do not help to restrict the one-dimensional variety of solutions of the analysis to an unique set of parameters.

The quotation of an "effective temperature" runs into conceptual difficulties in the case of extended atmospheres. The parameter $T_{*}(57.5 \mathrm{kK}$ for model A) follows simply from the luminosity and the reference radius $R_{*^{*}}$. Rosseland optical depths of $1,2 / 3$ or $1 / 3$ are reached at much larger radii: $2.5,3.3$ or $5.7 R_{*}$, respectively (model $A$ ). The corresponding "effective temperatures", if related to these radii, are about 37 , 32 , or $24 \mathrm{kK}$.

The luminosity of HD 50896 was determined to $\log (\mathrm{L} / \mathrm{L})=5.3$ (model $\mathrm{A})$, or less in the case of a smaller distance (cf. Table 1). A comparison with recent evolutionary calculations by Maeder and Meynet (1987) indicates an actual mass of about $10 \mathrm{M}$ (case A) from the (extrapolated) mass-luminosity relation for WR stars. But unfortunately, stars of this luminosity do not become WR stars, according to these evolutionary models, since the corresponding tracks (e.g. for an initial mass of $\left.20 \mathrm{M}_{\odot}\right)$ never return to the blue side of the HR diagram.

A more detailed version of this paper will be published in "Astronomy and Astrophysics".

\section{REFEREXCES}

Hamann, W.-R.: 1986, Astron. Astrophys. 160, 347

Hamann, W.-R.: 1987, in "Numerical Radiation Transfer", W. Kalkofen (ed.), Cambridge University Press (in press)

Hamann, w.-R., Schmutz, w.: 1987, Astron. Astrophys, 174, 173

Hillier, D.J.: 1987, Astrophys. J. Suppl. 63, 965 
Hogg, D.E.: 1982, in "Wolf-Rayet Stars: Observation, Physics, Evolution", IAU Symp. 99, C.W.H. deLoore and A.J. Willis (eds.), Reidel, Dordrecht, p. 221 Lundström, I., Stenholm, B.: 1984, Astron. Astrophys. Suppl. Ser. 58, 163 Maeder, A., Meynet, G.: 1987, Astron. Astrophys. (preprint)

Wessolowski, U., Schmutz, W., Hamann, W.-R.: 1987, Astron. Astrophys. (in press)
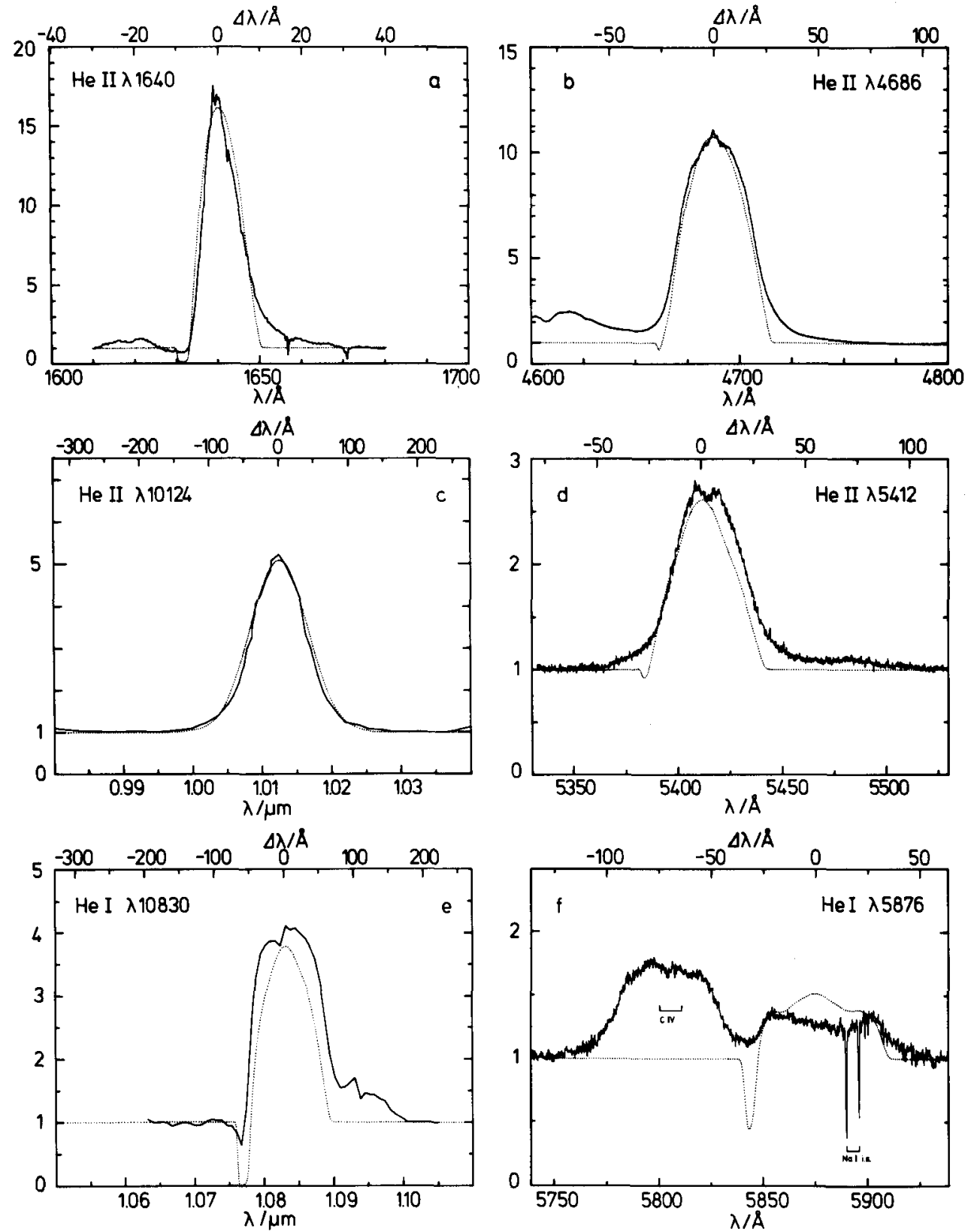

Fig. 1. Comparison of the observed line profiles to the theoretical profiles of model $C\left(\log \left(\dot{M} /\left(M_{\odot} / Y r\right)\right)=-4.7, R_{*}=1.5 R_{\odot}, T_{*}=63 \mathrm{kK}\right)$ 